\title{
Algebraic order and the Berezinskii-Kosterlitz-Thouless transition in an exciton-polariton gas
}

\author{
Wolfgang H. Nitsche, ${ }^{1, *}$ Na Young Kim, ${ }^{1}$ Georgios Roumpos, ${ }^{1, \dagger}$ Christian Schneider, ${ }^{2}$ Martin Kamp, ${ }^{2}$ Sven Höfling,,${ }^{2,3,4}$ \\ Alfred Forchel, ${ }^{2}$ and Yoshihisa Yamamoto ${ }^{1,4}$ \\ ${ }^{1}$ E. L. Ginzton Laboratory, Stanford University, Stanford, California 94305, USA \\ ${ }^{2}$ Technische Physik, Universität Würzburg, Am Hubland, 97074 Würzburg, Germany \\ ${ }^{3}$ School of Physics \& Astronomy, University of St Andrews, St Andrews, KY16 9SS, United Kingdom \\ ${ }^{4}$ National Institute of Informatics, Hitotsubashi, Chiyoda-ku, Tokyo 101-8430, Japan
}

(Received 27 December 2013; revised manuscript received 27 October 2014; published 24 November 2014)

\begin{abstract}
We observe quasi-long-range coherence in a two-dimensional condensate of exciton-polaritons. Our measurements confirm that the spatial correlation algebraically decays with a slow power law, whose exponent quantitatively behaves as predicted by the Berezinskii-Kosterlitz-Thouless theory. The exciton-polaritons are created by nonresonant optical pumping of a microcavity sample with embedded GaAs quantum wells at liquid helium temperature. Michelson interference is used to measure the coherence of the photons emitted by decaying exciton-polaritons.
\end{abstract}

DOI: 10.1103/PhysRevB.90.205430

PACS number(s): 71.35.Lk, 42.50.Gy, 73.20.Mf, 78.67.De

\section{INTRODUCTION}

Bose-Einstein condensation is characterized by the macroscopic occupation of the lowest-energy state and has been observed in atomic gases [1,2], as well as with quasiparticles in solid state systems [3,4]. It is accompanied by superfluidity [5-8] and long-range spatial coherence [9,10]. According to the Hohenberg-Mermin-Wagner theorem, true long-range order cannot exist in infinite uniform two-dimensional (2D) superfluids at nonzero temperatures [11,12]. However, in interacting systems, superfluidity can persist at nonzero temperatures in a state exhibiting quasi-long-range order characterized by an algebraic (power law) decay of the spatial correlation function. The transition from this state to the normal state is described by the Berezinskii-KosterlitzThouless (BKT) theory $[13,14]$ and is explained by the creation of free vortices that eliminate both the superfluidity and any quasi-long-range coherence [15]. In finite-sized systems, the quasi-long-range order can span the whole system, resembling true long-range order. Nevertheless, the transition from this state to the normal one is still expected to be of the BKT type. Previously, this transition has been demonstrated for superfluid liquid helium films [16,17], superconducting films [18,19], and 2D atomic gases [20], whereas spontaneous coherence of exciton-polaritons has been predicted [21] and observed [22] in the parametric regime. However, the power-law decay of spatial correlations expected for the BKT phase, which is its most distinct characteristic, has not been established quantitatively. In this paper, we show that the measured first-order spatial correlation function of an exciton-polariton condensate decays with a power law whose exponent is $\approx 1 / 4$

*Corresponding author: nitsche@stanford.edu; present address: Halliburton, Houston, Texas 77032, USA.

†Present address: Google, Mountain View, California 94043, USA.

Published by the American Physical Society under the terms of the Creative Commons Attribution 3.0 License. Further distribution of this work must maintain attribution to the author(s) and the published article's title, journal citation, and DOI. at the condensation threshold, as predicted by the BKT theory. A recent theoretical paper [23] confirmed that the continuous creation and decay of exciton-polaritons does not prevent our finite-sized nonequilibrium system from exhibiting the BKT state with quasi-long-range coherence.

\section{SPATIAL COHERENCE}

The first-order spatial correlation function

$$
g^{(1)}\left(\mathbf{r}_{1}, \mathbf{r}_{2}\right)=\frac{\left\langle\psi^{\dagger}\left(\mathbf{r}_{1}\right) \psi\left(\mathbf{r}_{2}\right)\right\rangle}{\sqrt{\left\langle\psi^{\dagger}\left(\mathbf{r}_{1}\right) \psi\left(\mathbf{r}_{1}\right)\right\rangle\left\langle\psi^{\dagger}\left(\mathbf{r}_{2}\right) \psi\left(\mathbf{r}_{2}\right)\right\rangle}}
$$

quantifies the coherence between points $\mathbf{r}_{1}$ and $\mathbf{r}_{2}$. Its values range from 0 in the case of no coherence to 1 for perfect coherence. In the case of 2D systems, coherence can be reduced by the thermal excitation of phononic long-wavelength phase fluctuations, as well as vortices.

Vortices are characterized by a vanishing superfluid density in their cores and a phase change of $2 \pi$ (or $-2 \pi$ in the case of an antivortex) around them. Large 2D superfluids always contain vortices, the presence of which decreases the free energy by increasing the entropy. These vortices can appear either in the form of bound vortex-antivortex pairs or as free vortices. The BKT theory predicts that the existence of free vortices is advantageous with respect to the free energy precisely if the 2D superfluid density $n_{\mathrm{s}}$ is less than the critical values of $4 / \lambda_{T}^{2}$, where $\lambda_{T}=h / \sqrt{2 \pi m_{\mathrm{eff}} k_{\mathrm{B}} T}$ is the thermal de Broglie wavelength $[13,14]$. This means that vortex-antivortex pairs unbind once the superfluid density drops below the critical value [15], either by decreasing $n_{\mathrm{s}}$ or increasing the temperature. Thus-created free vortices destroy the spatial coherence and the superfluid phase $[13,14]$ so that $n_{\mathrm{s}}$ must be either exactly zero or larger than the critical value. Therefore, a 2D superfluid with nonvanishing spatial coherence can only exist if $n_{\mathrm{s}}$ is above the critical value. In this case, the superfluid is in the BKT state, which means that vortices only exist in the form of bound vortex-antivortex pairs. These pairs do not affect the spatial coherence over large distances because the phase disturbance of each vortex is canceled out by its counterpart of antivortex. 

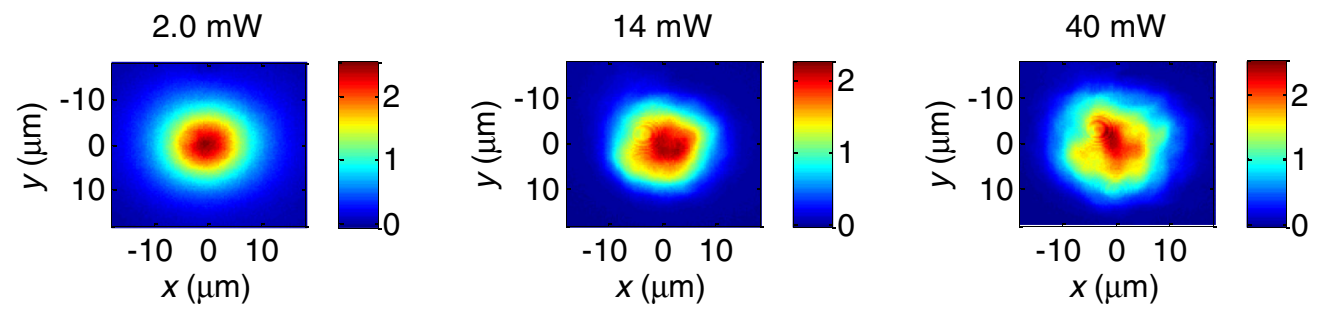

FIG. 1. (Color online) Pump-power-dependent density distribution. The irregular density profile at very high pump powers prevents us from extracting the exponent $a_{\mathrm{p}}$ in the very high-power regime (measured at $\delta \approx-1 \mathrm{meV}$ ).

The coherence over long distances in the BKT state is determined solely by the residual thermal excitation of phononic long-wavelength phase fluctuations. These phonons are predicted [15,24] to cause the coherence $g^{(1)}$ between $\mathbf{r}_{1}=(x ; y)$ and $\mathbf{r}_{2}=(-x ; y)$ to decay as a power law of the form

$$
g^{(1)}(x,-x)=\frac{n_{\mathrm{s}}}{n}\left(\frac{|x|}{\Lambda}\right)^{-a_{\mathrm{p}}},
$$

where $n_{\mathrm{s}}$ and $n$ are the superfluid and total densities, $\Lambda$ is a characteristic length on the order of the healing length $\xi$, and the exponent is $a_{\mathrm{p}}=1 /\left(n_{\mathrm{s}} \lambda_{T}^{2}\right)$. This power-law decay of $g^{(1)}$ is specifically a result of the density of states for phonons in a $2 \mathrm{D}$ system [15], and it differs distinctly from the three-dimensional Bose-Einstein condensate (BEC) state, where the correlation function approaches a constant value at large distances [25]. The critical density of $n_{\mathrm{s}}=4 / \lambda_{T}^{2}$ implies that $a_{\mathrm{p}}=1 / 4$ at the BKT threshold and $a_{\mathrm{p}}<1 / 4$ in the BKT state above the threshold, whereas below the threshold, no exponent $a_{\mathrm{p}}$ can be defined due to the absence of any long-range order.

\section{EXPERIMENTAL SETUP}

For our measurements, we use an exciton-polariton condensate for which it is possible to study the coherence properties for varying superfluid densities not only near the critical value but also far above that.

\section{A. Exciton-polaritons}

Exciton-polaritons are 2D bosonic quasiparticles, which can be described as the superposition of cavity photons and quantum well excitons [26]. At low temperatures, they exhibit dynamical condensation [3,4,27]. This condensate of exciton-polaritons can show a vortex-antivortex bound pair [28], as well as single vortices pinned at a defect [29-31]. (a)

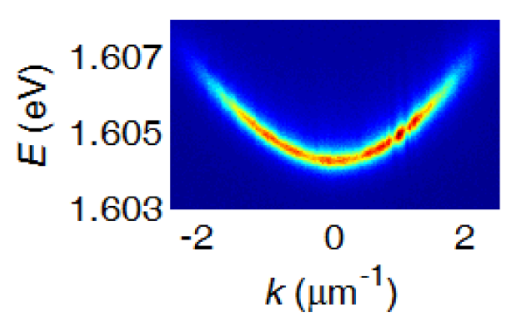

(b)

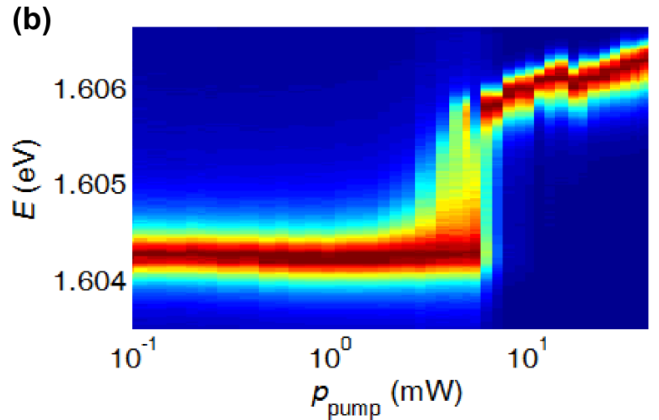

$6 \mathrm{~mW}$

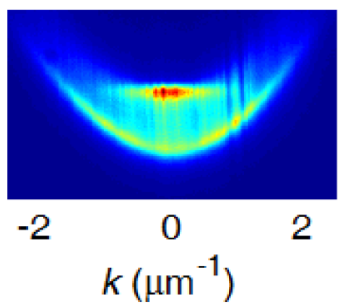

$40 \mathrm{~mW}$

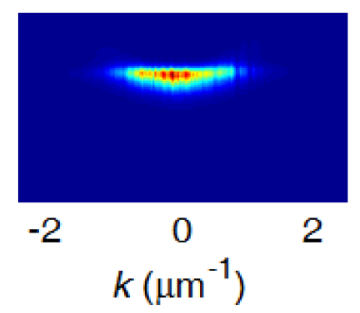

FIG. 2. (Color online) Blueshift of exciton-polariton energy. (a) Pump-power-dependent dispersion (measured at $\delta \approx-1 \mathrm{meV}$ ). Around $6 \mathrm{~mW}$, the condensation phase appears, and above this pump power, nearly all exciton-polaritons are in a ground state with zero momentum and one specific energy. A blueshift towards higher energies due to repulsive interaction between the exciton-polaritons is observed above the condensation threshold. (b) Normalized signal close to zero momentum at blue detuning of $\delta \approx-1$ meV. The measurement of the exciton-polariton density, described earlier, indicates that at the threshold of $p_{\text {pump }} \approx 6 \mathrm{~mW}$, the total exciton-polariton density is $n \approx 2 \mu \mathrm{m}^{-2}$, which corresponds to an exciton-polariton density per quantum well of $n_{\text {per Qw }} \approx 0.5 \mu \mathrm{m}^{-2}$. This small value confirms that we observe condensation rather than vertical cavity surface-emitting laser (VCSEL) lasing, because the latter requires significantly higher exciton-polariton densities of approximately $3 \times 10^{3} \mu \mathrm{m}^{-2}$ per quantum well to create a population inversion. (c) The same at red detuning of $\delta \approx 4$ meV. 

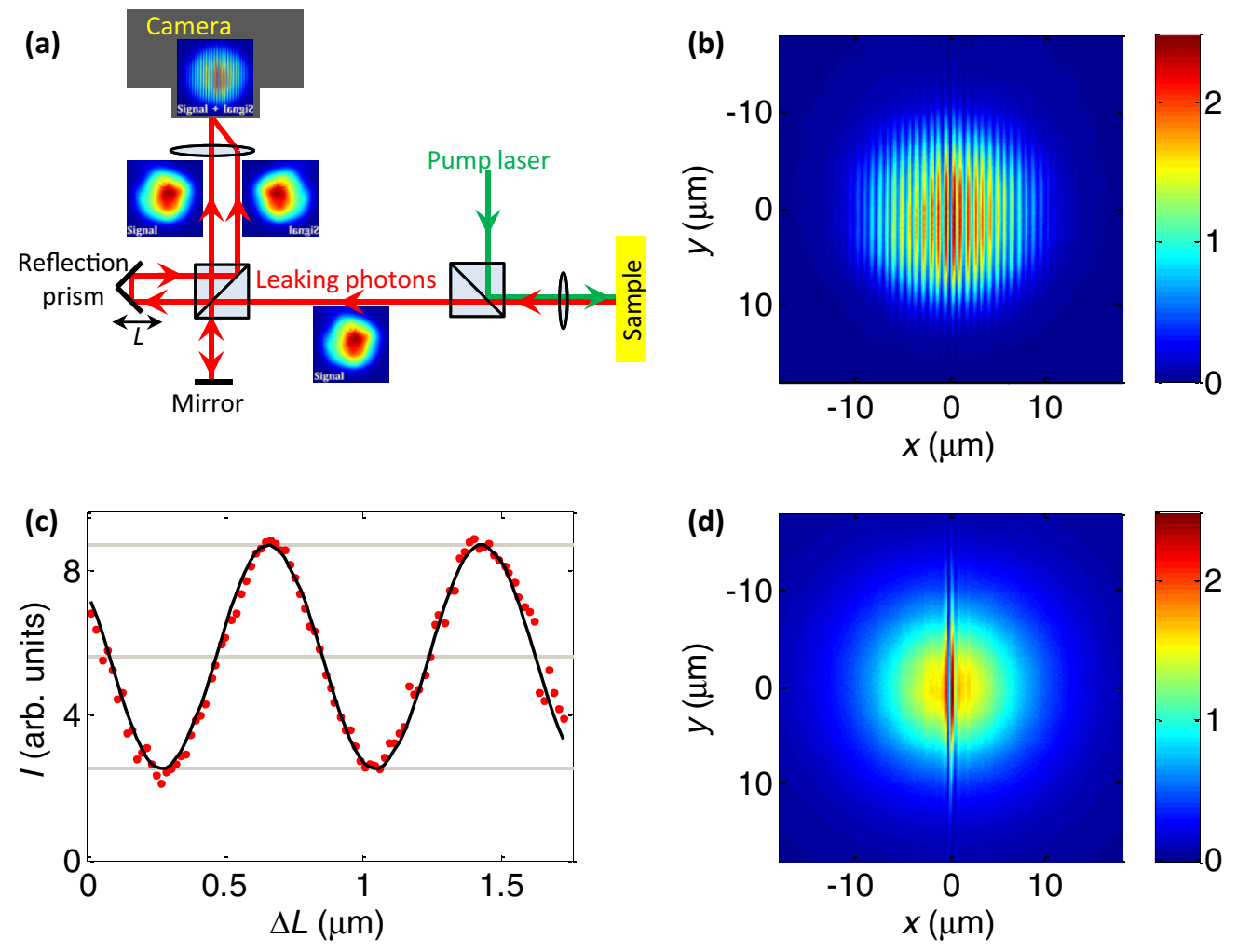

FIG. 3. (Color online) Michelson interference. (a) Michelson interferometer setup to measure the phase and the fringe visibility. (b) Measured interferogram (with intensity in arbitrary units) for one specific path-length difference $L$, measured at a pump power above the condensation threshold. (Same measurement as in the left column of Fig. 4) (c) The intensity of each pixel behaves like a sine function of the form $I_{\text {measured }}(\Delta L)=B+A \sin \left(w \Delta L-\varphi_{0}\right)$ if plotted as a function of the change $\Delta L$ of the path-length difference $L$. (d) Same as (b), but at a low pump power below the condensation threshold, where no spatial coherence exists over long distances. (See also Fig. 9(a) for the lack of coherence at low pump powers.)

Exciton-polaritons decay if a photon leaks out of the sample; in this case, the leaking photons preserve the energy, in-plane momentum, and coherence properties of the polaritons and the internal order parameter. Michelson interference allows us the measurement of $g^{(1)}$ of the leaking photons from the exciton-polariton condensate (see Fig. 3), which makes an exciton-polariton condensate a suitable system for studying the BKT physics.

\section{B. Sample}

The sample used in our measurements is grown on a GaAs wafer and consists of a $\lambda / 2$ AlAs cavity surrounded by Bragg reflectors with $20 \mathrm{AlAs} / \mathrm{AlGaAs}$ layer pairs at the top of the sample and 24 such pairs between the cavity and the substrate. Four 7-nm-thick GaAs quantum wells, separated from one another by 4-nm AlAs spacers, have been grown into the central antinode of the standing photon wave in the cavity. The $\lambda / 2$ cavity has been grown with a wedge form so that the detuning parameter $\delta=\left(\omega_{\text {cavity }}-\omega_{\text {exciton }}\right) \hbar$ changes with position. The splitting between the upper and the lower polaritons at zero detuning is approximately $9.6 \mathrm{meV}$, and the quality factor of the cavity mode (as measured in the far red detuned regime on the planar sample) is $Q_{\text {cavity }} \approx 3000$. The sample is installed in a helium flow cryostat that keeps the temperature at $5 \mathrm{~K}$.

\section{Creation of exciton-polaritons}

We create an exciton-polariton condensate (Figs. 1 and 2) by nonresonantly pumping the sample with perpendicular incidence by a Ti:sapphire laser in continuous-wave $(\mathrm{CW})$ operation. The pump wavelength is shorter than the cavity resonant wavelength, and is chosen to coincide with a reflection minimum outside the Bragg reflector stop band so that the pump light can efficiently reach the quantum wells, where it creates electron-hole pairs. To avoid thermal heating, the laser is chopped at $100 \mathrm{~Hz}$ with a duty cycle of $5 \%$ so that during each $10-\mu$ s period, the pump laser only hits the sample for $0.5 \mu \mathrm{s}$. This is still longer than the exciton-polariton lifetime, so quasi-CW excitation is guaranteed. In Fig. 2(a), one sees that the exciton-polaritons in the condensate are more energetic than those measured below the condensation threshold. Figure 2(b) shows the power-dependent signal that is emitted close to $k=0$. A jump to shorter wavelengths at the condensation threshold and a continuous blueshift above the threshold are the result of repulsive interaction between exciton-polaritons. The continuous blueshift above the threshold is a signature of exciton-polariton condensation, because it does not appear in the case of vertical-cavity surface-emitting laser (VCSEL) lasing.

For the measurements described in this paper, we use a laser beam with a Gaussian spatial intensity profile (with a full width at half maximum of $15 \mu \mathrm{m}$ ), which can only efficiently 

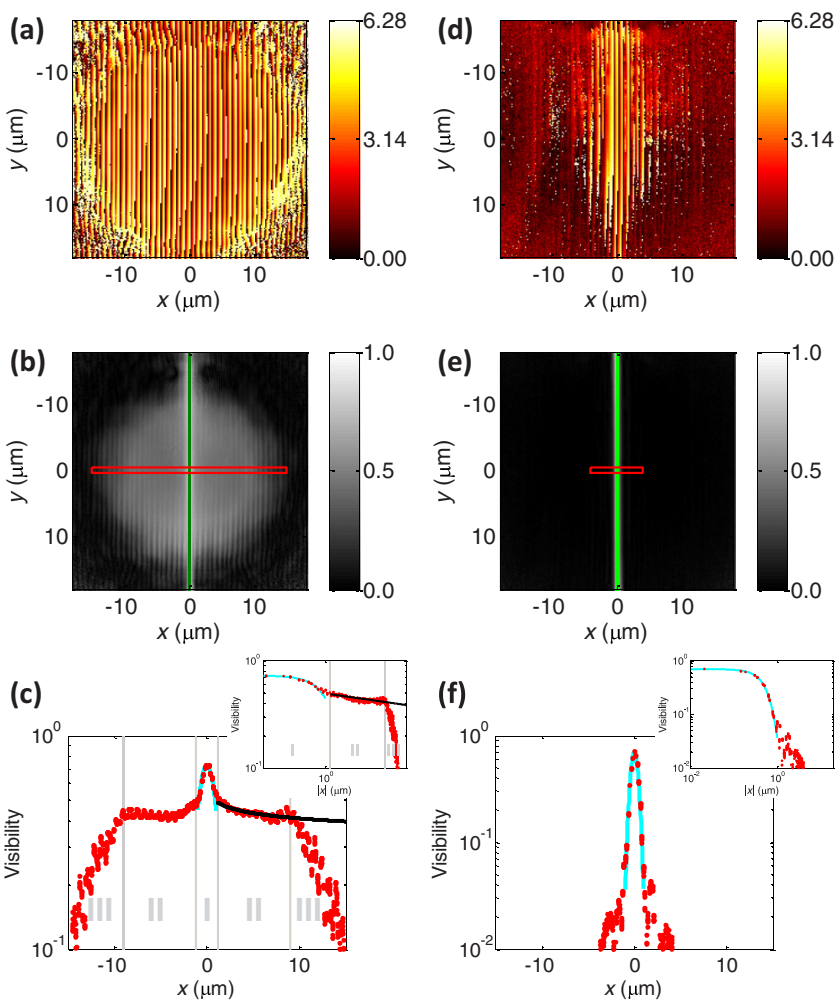

FIG. 4. (Color online) Measured phase and visibility (measured at detuning parameter $\delta \approx-1 \mathrm{meV}$ ). Left column: Above the condensation threshold (at $p_{\text {pump }}=15 \mathrm{~mW}$ ). (a) Phase map $\varphi_{0}$ produced by an ensemble of pixels. (b) Visibility map. The flip axis is shown as a line at $x=0$, and the region of interest used for further evaluation is highlighted by a rectangle. (c) The visibility within the region of interest as a function of the directed distance $x$ to the flip axis. In region II, a power-law fit with exponent $a_{\mathrm{p}}=0.082$ is shown. The regions I-III are selected manually. (Insert: The same in a log-log plot, where the power-law decay appears as a straight line.) Right column: The same characteristics as those below the condensation threshold (at $p_{\text {pump }}=2 \mathrm{~mW}$ ). Absence of quasi-long-range order is clearly seen in (d)-(f), and the fitted line in (f) is a Gaussian decay function.

excite the lowest spatial mode (with which it maximizes the overlap) of the condensate. Previous measurements [24] used a different sample and tried to create a uniform condensate density by using a top-hat-formed pump beam. However, such a top-hat pump profile can easily lead to condensate fragmentation into multiple spatio-energy modes [32]. In this case, the measured overall visibility might decay faster than the intrinsic coherence for each mode, because interference among different energy modes averages out to zero during the integration time of the camera [33].

\section{Michelson interference}

To measure the coherence of the emitted photons, we use a Michelson interferometer [Fig. 3(a)] with a variable path-length difference $L$ as described in Ref. [24]. A polarizing beam splitter is used to direct the pump beam onto the sample through an objective lens. The same objective lens collects the light emitted by the sample, and the component that is polarized perpendicular to the pump beam passes through the polarizing beam splitter and reaches the Michelson interference setup. There it is divided by a nonpolarizing beam splitter into the two arms of the interferometer. The light that travels through the first arm is reflected by a mirror and directed towards the camera, whereas the light in the other arm is reflected by a reflection prism, which flips the image along the $y$ axis before directing it to the camera. Therefore, at the camera, the light-which is emitted at point $(x ; y)$ of the sample and travels through the first arm-interferes with the light from point $(-x ; y)$ coming through the second arm. The wave fronts from the two arms reach the camera at a slightly different angle, which gives rise to interference fringes. Scattered light from the pump laser is filtered out by band-pass filters before reaching the camera. A piezo is used for changing the path-length difference by slightly moving the prism, and the interferogram is recorded for many path-length differences. The measured intensity of each of the pixels, corresponding to a position $( \pm x ; y)$ on the sample, as a function of the change $\Delta L$ of the path-length difference $L$ follows a sine law of the form

$$
I_{\text {measured }}(\Delta L)=B+A \sin \left(w \Delta L-\varphi_{0}\right) .
$$

The measured visibility $V=A / B$ [as shown in Fig. 4(b)] is therefore determined by the fitting parameters $A$ and $B$.

The visibility corresponding to $(x ; y)$ can be also calculated as

$$
V(x ; y)=\frac{2 \sqrt{I_{1}(x ; y) I_{2}(-x ; y)}}{I_{1}(x ; y)+I_{2}(-x ; y)} g^{(1)}(x ; y,-x ; y),
$$

where $I_{1}(x ; y)$ is the intensity of the signal that reaches the camera after being emitted from point $(x ; y)$ and traveling (a)

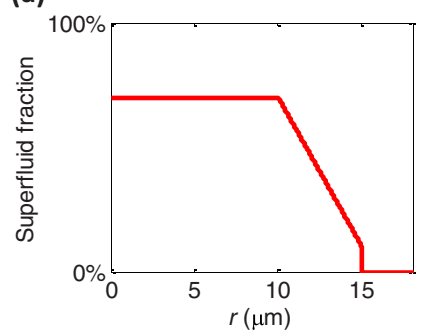

(b)

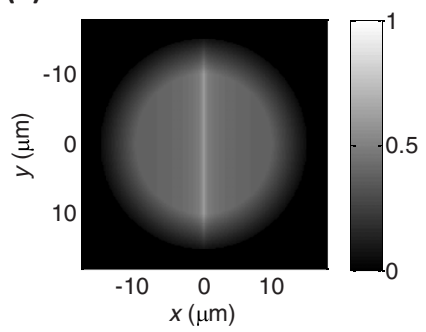

(c)

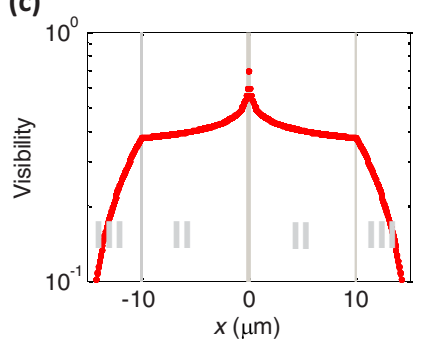

FIG. 5. (Color online) Simulated visibility. (a) Assumed position-dependent superfluid fraction as a function of radial position $r$. (b) Simulated visibility assuming $g^{(1)}=\left(n_{\mathrm{s}} / n\right)(|x| / 0.02 \mu \mathrm{m})^{-0.1}$. (c) Simulated visibility at $y \approx 0$. The simulated fast decay in region III shows that the fast decay in Fig. 4(c) can be explained by a decreasing superfluid fraction. 


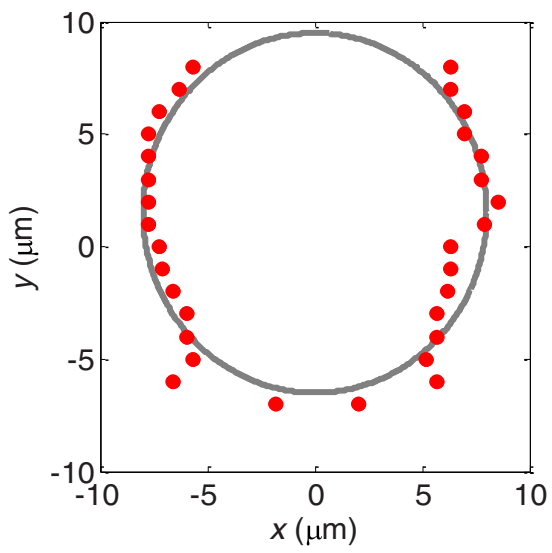

FIG. 6. (Color online) Transition from power law to fast decay of visibility. The dots show the cutoff points where the power law ends. They seem to form a circle that corresponds to the rotational symmetry of the Gaussian pump beam.

through arm 1 of the interferometer; likewise, $I_{2}$ travels through arm 2. With $I_{1}(x ; y)=f_{1} I(x ; y)$, where $I(x ; y)$ is the total emitted intensity at $(x ; y)$ and $f_{1}$ is the fraction traveling through arm 1 (and likewise for arm 2), the visibility becomes

$$
V(x)=\frac{2 \sqrt{f_{1} I(x) f_{2} I(-x)}}{f_{1} I(x)+f_{2} I(-x)} g^{(1)}(x,-x),
$$

where we omitted the constant $y$. In theory $f_{1}$ and $f_{2}$ should be identical, but in reality this is usually not the case, for example because the mirror in arm 1 has a different reflectivity than the reflection prism in arm 2 . In our setup, we have $f_{2} \approx 0.80 f_{1}$ (measured by individually blocking the arms), which for $I(x)=I(-x)$, as expected for a symmetrical condensate, gives $V(x)=0.994 g^{(1)}(x,-x)$. This means that the differences between $f_{1}$ and $f_{2}$ can be neglected. Assuming $f_{1} \approx f_{2}$ gives

$$
V(x)=\frac{2 \sqrt{I(x) I(-x)}}{I(x)+I(-x)} g^{(1)}(x,-x),
$$

implying that the visibility $V$ will decay faster than $g^{(1)}$ if $I(x) \neq I(-x)$ due to an irregular form of the condensate. For a perfectly symmetrical condensate with $I(x)=I(-x)$, we get

$$
V(x)=g^{(1)}(x,-x),
$$

which means that under optimal conditions, the measured visibility $V$ is identical to the correlation function $g^{(1)}$. In theory, Eq. (4) shows that $g^{(1)}$ could even be calculated without further approximations if $I_{1}(x ; y)$ and $I_{2}(x ; y)$ are individually recorded by using only the respective arm of the interferometer while blocking the other arm. However, under realistic experimental conditions, the exact form of a nonsymmetrical condensate changes with time, which means that the intensities that are measured while one of the interferometer arms is blocked are not necessarily identical to the intensities traveling through the individual arms during the interference measurement.

The sine fit [Eq. (3)] also gives the phase $\varphi_{0}$ [Fig. 4(a)], which can be used to confirm that the long-range order is produced and the fit is able to extract reliable data of the visibility [24]. (a)

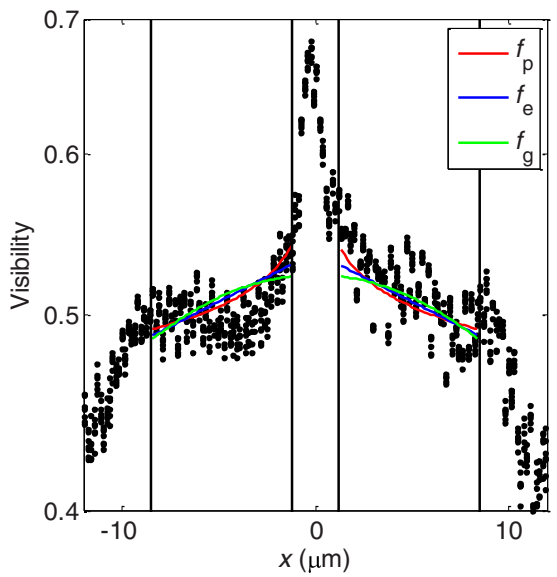

(b)

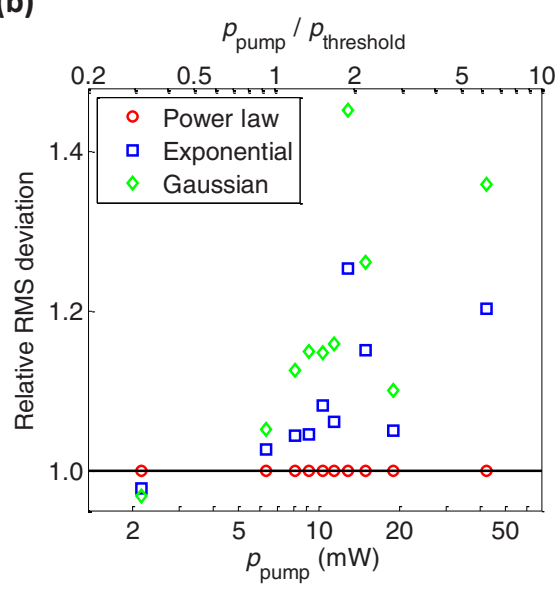

(c)

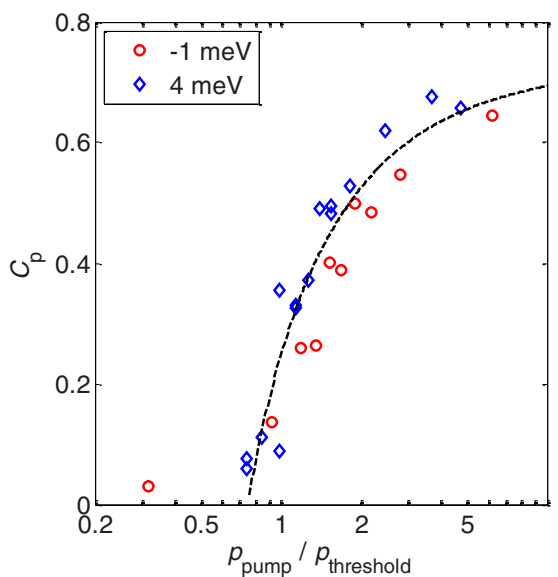

FIG. 7. (Color online) Fitting various decay functions to the visibility data. (a) The used decay functions are a power law of the form $f_{\mathrm{p}}(x)=c_{\mathrm{p}}|x / \mu \mathrm{m}|^{-a_{\mathrm{p}}}$, exponential function $f_{\mathrm{e}}(x)=c_{\mathrm{e}} \exp \left(-a_{\mathrm{e}}|x / \mu \mathrm{m}|\right)$, and Gaussian function $f_{\mathrm{g}}(x)=c_{\mathrm{g}} \exp \left[-a_{\mathrm{g}}(x / \mu \mathrm{m})^{2}\right]$. The fits have been performed by minimizing the RMS deviation $D_{i}=\sqrt{\sum_{n}\left[f_{i}\left(x_{n}\right)-V_{n}\right]^{2} / N}$ between the fitted functions $f_{i}$ (with $i \in\{\mathrm{p}, \mathrm{e}, \mathrm{g}\}$ ) and the $N$ measured positions and visibilities $\left(x_{n} ; V_{n}\right)$ with $x_{\min }<\left|x_{n}\right|<x_{\max }$, where the limits of the intermediate range $x_{\min }$ and $x_{\max }$ (marked by black vertical lines) have been chosen manually. We use the same fit for the $x<0$ and the $x>0$ regions. The visibility shown here has been measured at $\delta \approx-1 \mathrm{meV}$ and $p_{\text {pump }}=19 \mathrm{~mW}$. (b) The relative RMS deviations $R_{i}=\frac{D_{i}}{D_{\mathrm{p}}}$ measured at $\delta \approx-1 \mathrm{meV}$. Above the condensation threshold, we always observed $D_{\mathrm{p}}<D_{\mathrm{e}}<D_{\mathrm{g}}$, which means that the power-law decay gives the best match and the Gaussian fit gives the worst match. (c) The pump power dependence of the factors $c_{\mathrm{p}}$ determined by this fit at $\delta \approx-1 \mathrm{meV}$ and at $\delta \approx 4 \mathrm{meV}$. The dashed line is introduced to guide the eye. 


\section{MEASURED COHERENCE AND ITS INTERPRETATION}

A typical result [Fig. 4(a)-4(c)] for the measured phase and fringe visibility shows three distinct regions: The visibility over short distances (region I) decays according to a Gaussian law. This is similar to the correlation function decay of a Bose gas in thermal equilibrium [25], where the width of the Gaussian is proportional to the de Broglie wavelength. However, in our system, the population of energetically higher modes depends primarily on their overlap with the Gaussian pump profile; therefore, we cannot deduce an accurate temperature from this Gaussian width [24].

In the region of intermediate distances (region II), the decay of the visibility follows a power law as theoretically predicted for the BKT state. From this, we determine the exponent $a_{\mathrm{p}}$ by fitting a power law proportional to $|x|^{-a_{\mathrm{p}}}$ to the measured fringe visibility in this region. For even larger distances (region III), the visibility starts to decay faster, which we attribute to the decrease of the superfluid fraction towards the edge of the condensate. The intensity of the Gaussian pump decreases towards the edge, which is expected to lead to a decrease of the superfluid fraction $n_{\mathrm{s}} / n$. According to Eq. (2) (and as
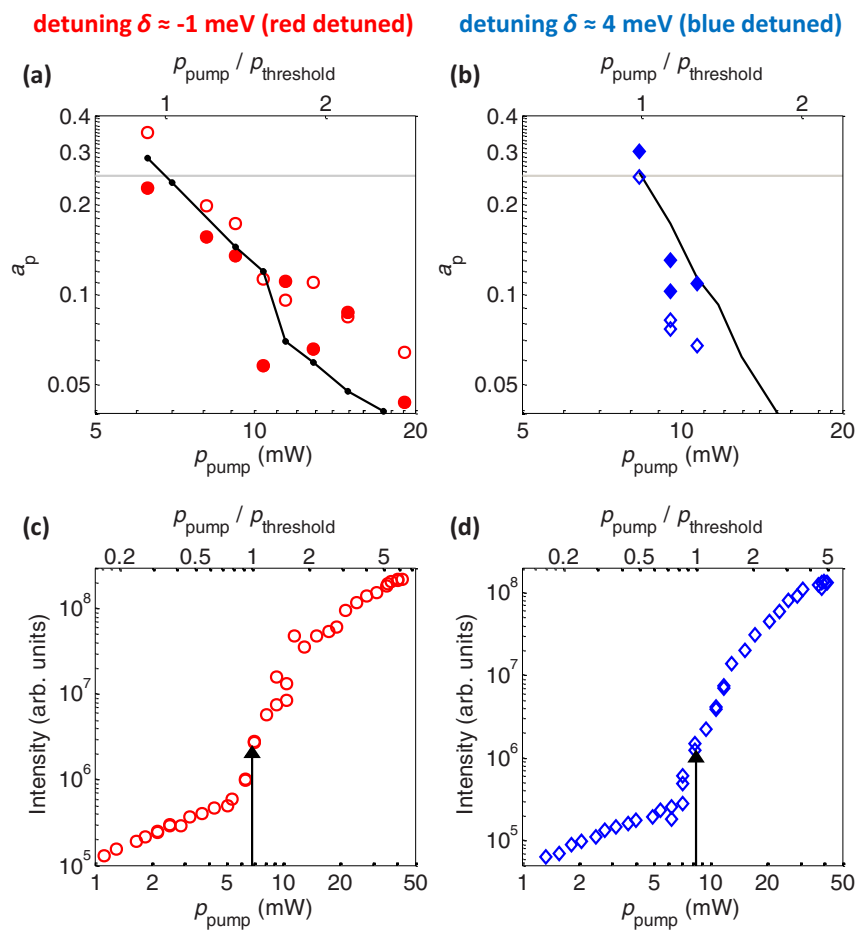

FIG. 8. (Color online) Pump-power dependence. Upper row: Pump-power dependence of the exponent $a_{\mathrm{p}}$ for two detuning values. The symbols show the measured exponents as determined by a power-law fit as shown in Fig. 4(c). The gray horizontal line at 0.25 shows the theoretically predicted exponent at the threshold. The black continuous line shows the estimated inverse superfluid phase-space density $1 /\left(n_{\mathrm{s}} \lambda_{T}^{2}\right)$, and as predicted by the theory, it matches the measured exponents $a_{\mathrm{p}}$. Filled symbols correspond to the $x<0$ region. Lower row: Pump-power-dependent peak intensity, as determined by an energy and momentum resolved measurement. The threshold pump powers defined by the critical exponent $a_{\mathrm{p}}=0.25$ (upper traces) are indicated by the black arrows. shown in Fig. 5), this picture can explain the fast drop of $g^{(1)}$. To demonstrate this, we simulated the visibility (Fig. 5) by assuming a condensate with a radius of $15 \mu \mathrm{m}$, where the superfluid fraction is constant for $r<10 \mu \mathrm{m}$ and slowly decreases to a small value at $r=15 \mu \mathrm{m}$, where it jumps to 0\%. In Fig. 4(c), we see that the power law (region II) ends at a "cutoff" length beyond which a faster decay (region III) is observed. These data have been extracted close to $y=0$ in Fig. 4(b). By performing the same evaluation for different $y$ values, we see that the cutoff points seem to lie on a circle (Fig. 6), which confirms our interpretation that the fast decay of the visibility is caused by a decrease of the condensation fraction towards the edge of the condensate.

In Fig. 7, three decay functions are tested against the measured visibility. The power-law decay function gives the best match after the individual minimization of the root-meansquare (RMS) deviation, which strongly indicates that the coherence decays with a power law, as theoretically predicted.
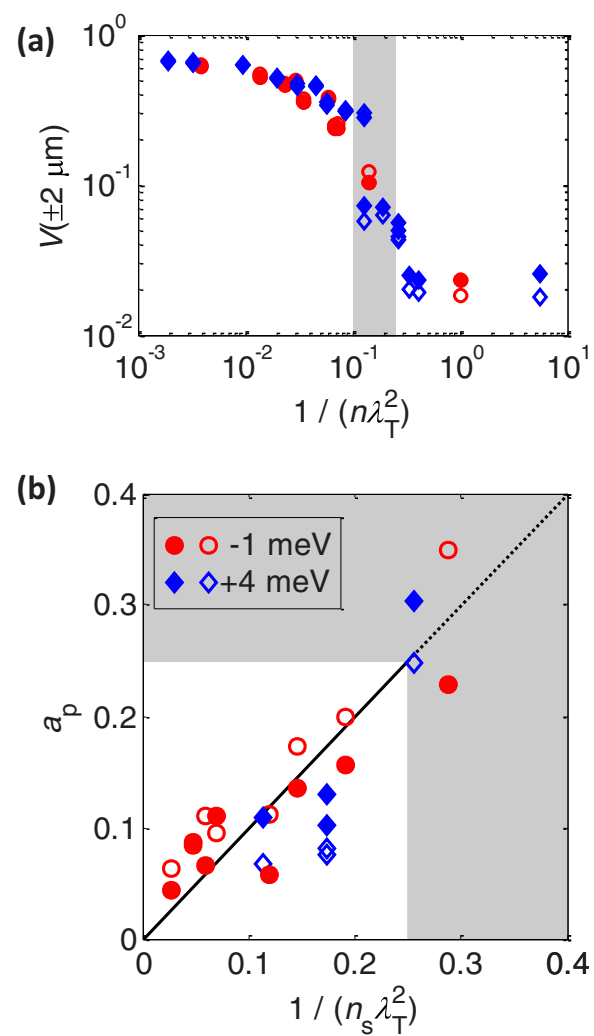

FIG. 9. (Color online) Phase-space density, visibility, and powerlaw exponent. As in Fig. 8, filled symbols correspond to the $x<0$ region, and different kinds of symbols correspond to different detunings. (a) The visibility at $x= \pm 2 \mu \mathrm{m}$ versus the inverse phase-space density $1 /\left(n \lambda_{T}^{2}\right)$. The threshold is upper bounded by $1 /\left(n_{\mathrm{s}} \lambda_{T}^{2}\right)=1 / 4$ and lower bounded [34] by $1 / \ln \left[380 \hbar^{2} /\left(m_{\text {eff }} g_{\text {interaction }}\right)\right]=1 / 10$, which are shown by the shaded area. (b) Measured exponents $a_{\mathrm{p}}$ as a function of estimated inverse superfluid phase-space density $1 /\left(n_{\mathrm{s}} \lambda_{T}^{2}\right)$. The black line shows the predicted $a_{\mathrm{p}}^{\text {calculated }}=1 /\left(n_{\mathrm{s}} \lambda_{T}^{2}\right)$, and the region above the expected threshold of 0.25 is shaded in gray. Although we did not change the temperature during the measurement, the abscissa can be interpreted as the dimensionless temperature (in units of $2 \pi m_{\text {eff }}^{-1} k_{\mathrm{B}}^{-1} n_{\mathrm{s}} \hbar^{2}$ ), and the BKT phase transition occurs at the dimensionless temperature of 0.25 in these units. 

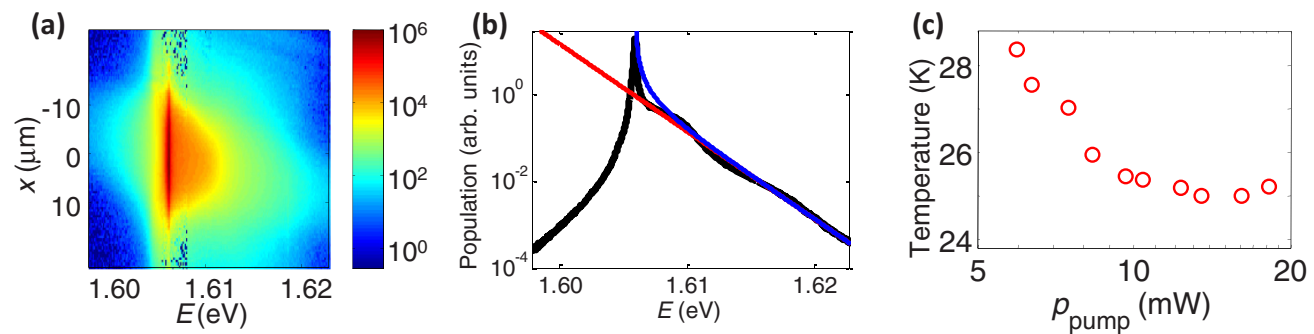

FIG. 10. (Color online) Determination of the exciton-polariton temperature. (a) Position $x$ and energy $E$ resolved intensity. (b) Measured population (black) with Boltzmann (straight line) and Bose-Einstein distributions for $25 \mathrm{~K}$. (c) Effective temperatures determined by fitting a thermal distribution to the population (performed for different pump powers in the case of a detuning of $\delta \approx-1 \mathrm{meV}$ ).

A pump-power-dependent measurement of the fringe visibility $\quad a_{\mathrm{p}}$ is approximately $1 / 4$ at the threshold and decreases with shows that, as predicted by the BKT theory, the exponent increasing pump power (Fig. 8). This figure also shows that
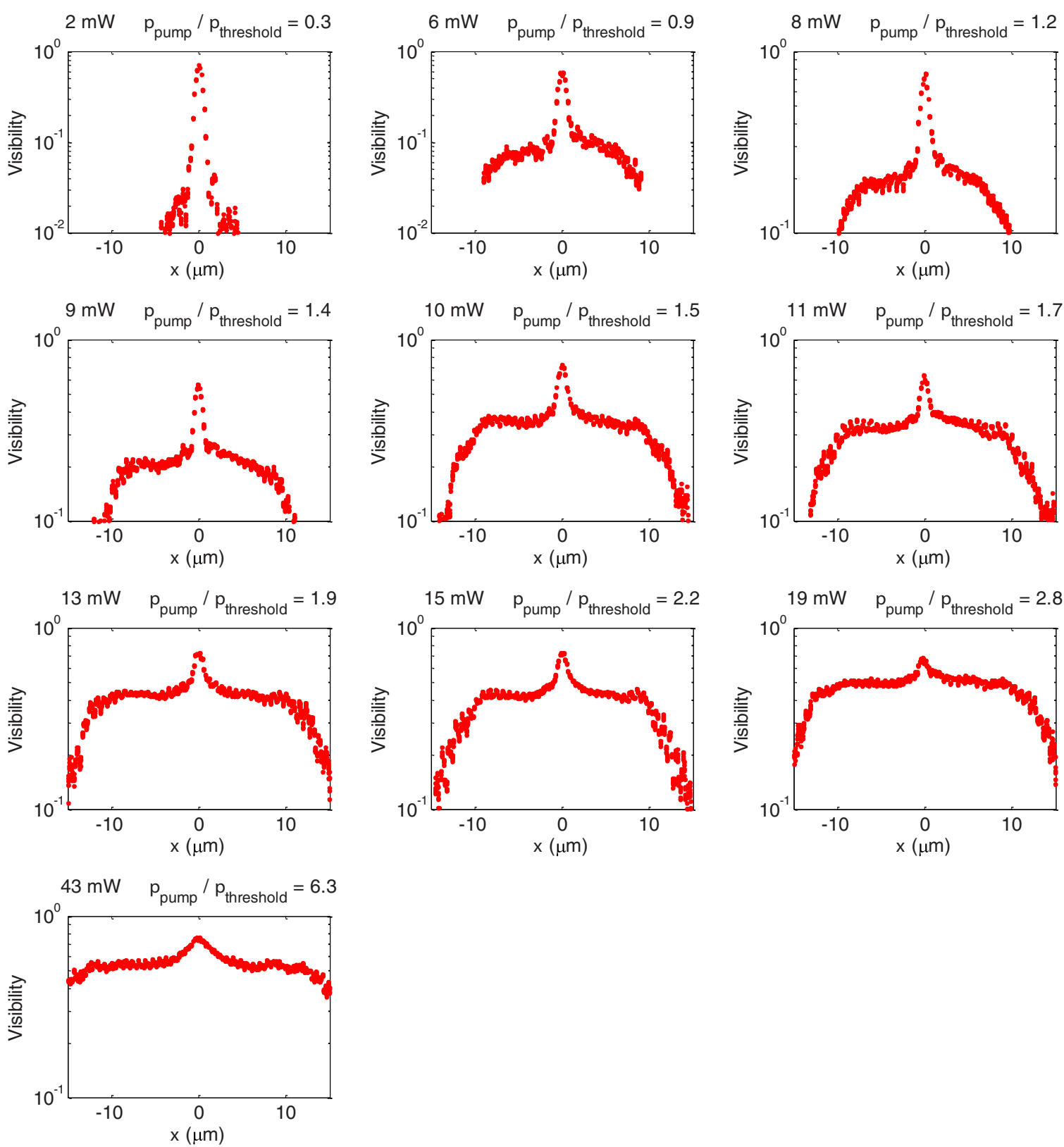

FIG. 11. (Color online) Measured visibility at a detuning of $-1 \mathrm{meV}$. One can see the existence of quasi-long-range order above the condensation threshold but only Gaussian decay below the condensation threshold. 
overall, the exponents $a_{\mathrm{p}}$ extracted for both $x>0$ and $x<0$ are equivalent, even if each measurement of the visibility [as shown in Fig. 4(c)] appears slightly asymmetrical due to experimental imperfections. As shown in Fig. 8, for pump powers moderately above the condensation threshold, increasing the pump power decreases the exponent $a_{\mathrm{p}}$ of the power law. For even higher pump powers, the measured $a_{\mathrm{p}}$ seems to increase instead of showing the expected steady decrease. This increase can be explained as an artifact resulting from the spatial density of the condensate, which at high pump powers deviates from a Gaussian profile and becomes increasingly nonsymmetrical (Fig. 1). Thus, $I(x) \neq I(-x)$,
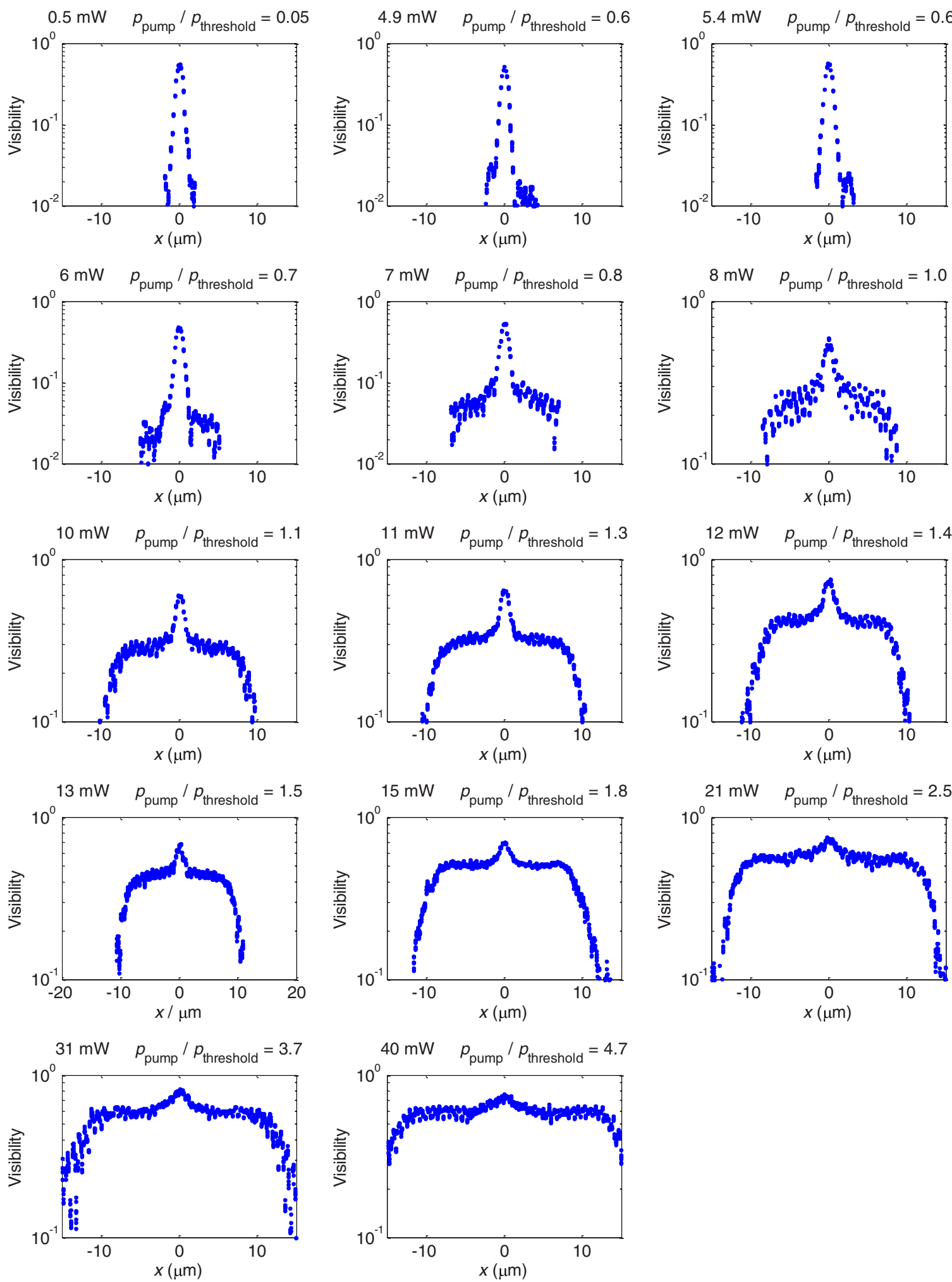

FIG. 12. (Color online) Measured visibility at a detuning of $4 \mathrm{meV}$. 
which causes the measured visibility to decay faster than $g^{(1)}$. Therefore, for Fig. 8 (upper row) and Fig. 9(b), we did not consider data from very high pump powers corresponding to an irregular condensate form.

We believe that the irregularities of the condensate form are caused by local fluctuations of the sample properties. In the case of irregular condensate forms, we see certain patterns like dark lines going through the condensate. If we slightly move the sample, these patterns move with the sample, which indicates that they are caused by local properties of the sample. For actual measurements, we always carefully chose spots on the sample where the irregularities are minimized, which means the measurements have been performed at positions where the sample properties are believed to be nearly constant throughout the area of the condensate. In theory, the visibility is always expected to be symmetrical so that $V(x)=V(-x)$. However, Eq. (5) shows that under realistic experimental conditions, $V(x) \neq V(-x)$ is possible if $I(x) \neq I(-x)$ and at the same time $f_{1} \neq f_{2}$. This can explain the slightly asymmetrical data in Fig. 4(c).

By recording the position resolved image (Fig. 1) of the condensate with a calibrated camera and estimating the transmittance $T_{\text {path }}$ of the beam path from the sample to the camera, we can estimate the photon flux $j$ at which photons leak out of the sample. The exciton-polariton lifetime $\tau_{\text {exciton-polariton }}$ can be calculated as $\tau_{\text {exciton-polariton }} \approx \tau_{\text {cavity }} /|C|^{2}$, where the Hopfield coefficient $|C|^{2}$ is the detuning-dependent photon component of the exciton-polariton and the photon lifetime $\tau_{\text {cavity }}$ is known from the quality factor of the cavity. The $2 \mathrm{D}$ total exciton-polariton density is estimated as $n=j \tau_{\text {exciton-polariton. We assume that above the threshold, }}$ the superfluid density $n_{\mathrm{s}}$ is always about half of this total density $n$, because the measured visibility is approximately $1 / 2$ at the transition from region I to region II in Fig. 4(c). While the sample is kept at $5 \mathrm{~K}$, exciton-polaritons, which are not at thermal equilibrium with the lattice, have a different temperature. By fitting a thermal distribution to the measured energy resolved population (Fig. 10), although the system is not in thermal equilibrium, we can estimated an effective temperature of the exciton-polaritons to be always $\approx 25 \mathrm{~K}$. From this, we get the thermal de Broglie wavelength $\lambda_{T}$. The continuous black line in Fig. 8 shows the calculated value for $a_{\mathrm{p}}^{\text {calculated }}:=1 /\left(n_{\mathrm{s}} \lambda_{T}^{2}\right)$ using these experimental values of $n_{\mathrm{s}}$ and $\lambda_{T}$, and it gives a reasonable match with the experimental values for $a_{\mathrm{p}}$ from the interference measurements.

We show one-to-one correspondence [Fig. 9(b)] between the measured exponents $a_{\mathrm{p}}$ and the phase-space density of $1 /\left(n_{\mathrm{s}} \lambda_{T}^{2}\right)$, as well as the predicted continuous line with $a_{\mathrm{p}}^{\text {calculated }}=1 /\left(n_{\mathrm{s}} \lambda_{T}^{2}\right)$. The exponents $a_{\mathrm{p}}$, which have been measured for different pump powers and detuning parameters (Figs. 11 and 12), follow the predicted universal line. This observation indicates that the 2D exciton-polariton condensate behaves as expected from the BKT theory.

\section{SIMULATED POWER LAW}

The theory for the BKT phase predicts that the power-law decay of the correlation function $g^{(1)}$ is the result of thermally excited phonon modes, whereas all vortices are paired so that they cannot affect $g^{(1)}$. We simulated a 2D condensate whose
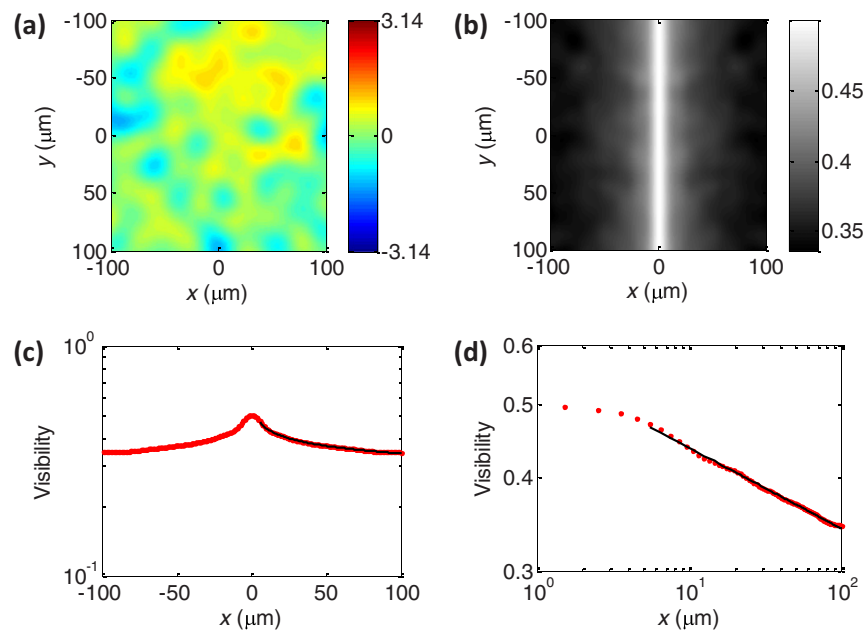

FIG. 13. (Color online) Simulated power law. (a) Phase $\Theta$ in one run of the simulation. (b) and (c) Visibility averaged over 1000 runs of the simulation. (d) The same visibility shown as a log-log plot, where the power law appears as a straight line.

phase fluctuations are caused by phonons with wavelengths of at least $2 \pi$ times the healing length (Fig. 13). For this simulation, we assumed that the number of phonons in the different possible modes follow a phonon statistics, meaning that the probability to have exactly $N_{\mathbf{k}}$ phonons in the mode $\mathbf{k}$ is

$$
p_{N_{\mathbf{k}}}=\left[1-\exp \left(-\frac{\varepsilon_{\mathbf{k}}}{k_{\mathrm{B}} T}\right)\right] \exp \left(-\frac{N_{\mathbf{k}} \varepsilon_{\mathbf{k}}}{k_{\mathrm{B}} T}\right),
$$

where $\varepsilon_{\mathbf{k}}=\hbar c|\mathbf{k}|$ is the energy per phonon in this mode $\mathbf{k}$. The local phase can be expressed [15] in the form $\Theta(\mathbf{r})=$ $\alpha_{\mathbf{k}} \sin \left(\mathbf{k r}+\varphi_{\mathbf{k}}\right)$, where the Fourier amplitudes $\alpha_{\mathbf{k}}$ depend on the numbers $N_{\mathbf{k}}$ of phonons in the respective modes and where a 2D constant density of states applies. No vortices have been considered in this simulation. As expected, the simulated visibility decays with a power law, which confirms that this characteristic decay can be caused by thermally excited phonons, rather than by the presence of vortices. As shown in Ref. [15], the same result can also be derived analytically.

\section{CONCLUSION}

Our measurements confirm that exciton-polariton condensation can be understood not by the BEC model but exclusively within the BKT model. The measured first-order spatial coherence function fully supports a unique $2 \mathrm{D}$ superfluid characteristic both at and above the threshold.

\section{ACKNOWLEDGMENTS}

The authors acknowledge helpful discussions with E. Altman, J. Dalibard, M. Fraser, J. Keeling, and W. Phillips. This research has been supported by the Japan Society for the Promotion of Science (JSPS) through its Funding Program for World-Leading Innovative Research and Development on Science and Technology (FIRST Program), by the U.S. Navy/SPAWAR Grant No. N66001-09-1-2024, and by the National Science Foundation Grant No. ECCS-09 25549. W.H.N. acknowledges the Gerhard Casper Stanford Graduate Fellowship. 
[1] M. H. Anderson, J. R. Ensher, M. R. Matthews, C. E. Wieman, and E. A. Cornell, Science 269, 198 (1995).

[2] K. B. Davis, M.-O. Mewes, M. R. Andrews, N. J. van Druten, D. S. Durfee, D. M. Kurn, and W. Ketterle, Phys. Rev. Lett. 75, 3969 (1995).

[3] H. Deng, G. Weihs, C. Santori, C. Bloch, and Y. Yamamoto, Science 298, 199 (2002).

[4] J. Kasprzak, M. Richard, S. Kundermann, A. Baas, P. Jeambrun, J. M. J. Keeling, F. M. Marchetti, M. H. Szymańska, R. André, J. L. Staehli, V. Savona, P. B. Littlewood, B. Deveaud, and L. S. Dang, Nature (London) 443, 409 (2006).

[5] P. Kapitza, Nature (London) 141, 74 (1938).

[6] A. Amo, D. Sanvitto, F. P. Laussy, D. Ballarini, E. del Valle, M. D. Martin, A. Lemaître, J. Bloch, D. N. Krizhanovskii, M. S. Skolnick, C. Tejedor, and L. Viña, Nature (London) 457, 291 (2009).

[7] A. Amo, J. Lefrère, S. Pigeon, C. Adrados, C. Ciuti, I. Carusotto, R. Houdré, E. Giacobino, and A. Bramati, Nat. Phys. 5, 805 (2009).

[8] D. Sanvitto, F. M. Marchetti, M. H. Szymańska, G. Tosi, M. Baudisch, F. P. Laussy, D. N. Krizhanovskii, M. S. Skolnick, L. Marrucci, A. Lemaître, J. Bloch, C. Tejedor, and L. Viña, Nat. Phys. 6, 527 (2010).

[9] H. Deng, G. S. Solomon, R. Hey, K. H. Ploog, and Y. Yamamoto, Phys. Rev. Lett. 99, 126403 (2007).

[10] F. Manni, K. G. Lagoudakis, B. Pietka, L. Fontanesi, M. Wouters, V. Savona, R. André, and B. Deveaud-Plédran, Phys. Rev. Lett. 106, 176401 (2011).

[11] N. D. Mermin and H. Wagner, Phys. Rev. Lett. 17, 1133 (1966).

[12] P. C. Hohenberg, Phys. Rev. 158, 383 (1967).

[13] V. L. Berezinskiǔ, Zh. Eksp. Teor. Fiz. 61, 1144 (1971) [Sov. Phys. JETP 34, 610 (1972)].

[14] J. M. Kosterlitz and D. J. Thouless, J. Phys. C 6, 1181 (1973).

[15] Z. Hadzibabic and J. Dalibard, Riv. Nuovo Cimento 34, 389 (2011).

[16] D. J. Bishop and J. D. Reppy, Phys. Rev. Lett. 40, 1727 (1978).

[17] D. J. Bishop and J. D. Reppy, Phys. Rev. B 22, 5171 (1980).
[18] M. R. Beasley, J. E. Mooij, and T. P. Orlando, Phys. Rev. Lett. 42, 1165 (1979).

[19] A. F. Hebard and A. T. Fiory, Phys. Rev. Lett. 44, 291 (1980).

[20] Z. Hadzibabic, P. Krüger, M. Cheneau, B. Battelier, and J. Dalibard, Nature (London) 441, 1118 (2006).

[21] I. Carusotto and C. Ciuti, Phys. Rev. B 72, 125335 (2005).

[22] R. Spano, J. Cuadra, G. Tosi, C. Antón, C. A. Lingg, D. Sanvitto, M. D. Martín, L. Viña, P. R. Eastham, M. van der Poel, and J. M. Hvam, New J. Phys. 14, 075018 (2012).

[23] E. Altman, L. M. Sieberer, L. Chen, S. Diehl, and J. Toner, arXiv:1311.0876.

[24] G. Roumpos, M. Lohse, W. H. Nitsche, J. Keeling, M. H. Szymańska, P. B. Littlewood, A. Löffler, S. Höfling, L. Worschech, A. Forchel, and Y. Yamamoto, Proc. Natl. Acad. Sci. USA 109, 6467 (2012).

[25] I. Bloch, T. W. Hänsch, and T. Esslinger, Nature (London) 403, 166 (2000).

[26] C. Weisbuch, M. Nishioka, A. Ishikawa, and Y. Arakawa, Phys. Rev. Lett. 69, 3314 (1992).

[27] R. Balili, V. Hartwell, D. Snoke, L. Pfeiffer, and K. West, Science 316, 1007 (2007).

[28] G. Roumpos, M. D. Fraser, A. Löffler, S. Höfling, A. Forchel, and Y. Yamamoto, Nat. Phys. 7, 129 (2011).

[29] K. G. Lagoudakis, M. Wouters, M. Richard, A. Baas, I. Carusotto, R. André, L. S. Dang, and B. Deveaud-Plédran, Nat. Phys. 4, 706 (2008).

[30] K. G. Lagoudakis, T. Ostatnický, A. V. Kavokin, Y. G. Rubo, R André, and B. Deveaud-Plédran, Science 326, 974 (2009).

[31] K. G. Lagoudakis, F. Manni, B. Pietka, M. Wouters, T. C. H. Liew, V. Savona, A. V. Kavokin, R. André, and B. DeveaudPlédran, Phys. Rev. Lett. 106, 115301 (2011).

[32] G. Roumpos, W. H. Nitsche, S. Höfling, A. Forchel, and Y. Yamamoto, Phys. Rev. Lett. 104, 126403 (2010).

[33] W. H. Nitsche, Ph.D. Thesis, Stanford University, 2014, http://purl.stanford.edu/by756rx2856.

[34] N. Prokof'ev, O. Ruebenacker, and B. Svistunov, Phys. Rev. Lett. 87, 270402 (2001). 